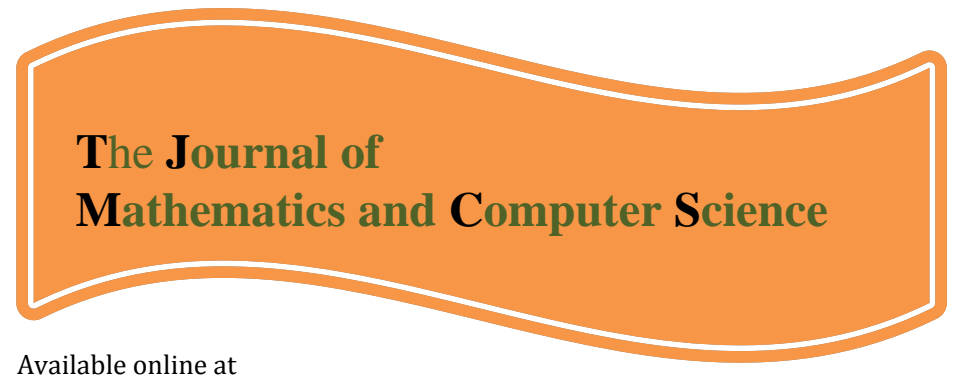

http://www.TJMCS.com

The Journal of Mathematics and Computer Science Vol .2 No.3 (2011) 453-458

\title{
Adjoint Operator in Fuzzy Normed Linear Spaces
}

\author{
Ali Taghavi ${ }^{1}$ and Majid Mehdizadeh ${ }^{2, *}$ \\ ${ }^{1}$ Department of Mathematics, Faculty of Mathematical Sciences Universuty of Mazandaran, Iran \\ E-mail address: Taghavi@nit.ac.ir \\ 2 Young Researchers Club, Islamic Azad University, Ghaemshahr, Iran \\ *E-mail address: mehdizadeh_77216@yahoo.com
}

Received: August 2010, Revised: November 2010

Online Publication: January 2011

\begin{abstract}
In this paper, the definition adjoint of the operator on fuzzy normed linear spaces is introduced. It is shown that if $(X,\|\|)$ and $\left(Y,\|\|^{\sim}\right)$ are two fuzzy normed linear spaces and $T: X \rightarrow Y$ be a strongly (weakly) fuzzy bounded linear operator, then $T^{*}: Y^{*} \rightarrow X^{*}$ (adjoint of $T$ ) is strongly ( weakly) fuzzy bounded linear operator and $\|T\|_{\alpha}^{*}=\left\|T^{*}\right\|_{\alpha}^{*}$, for each $\alpha \in(0,1]$.
\end{abstract}

Keywords: Adjoint operator; Dual space; Fuzzy linear operator; Fuzzy norm.

\section{Introduction}

In 1984, Katsaras [7], first introduced the notion of fuzzy norm on a linear space. In 1992, Felbin [4] introduced an idea of a fuzzy norm on a linear space by assigning a fuzzy real number to each element of the linear space so that the corresponding metric associated this fuzzy norm is of Kaleva type [6] fuzzy metric. She also introduced an idea of fuzzy bounded linear operator, the norm of which is a fuzzy number. In 1994, Cheng and Mordeson [3] introduced another idea of a fuzzy norm on a linear space in such a manner that the corresponding fuzzy metric of it is of Kramosil and Michelek type [8]. 
Considering fuzzy functions, they defined boundedness of fuzzy linear operator. Very recently Xiao and Zhu [9] redefined, in a more general setting, the idea of Felbin's [4] definition of fuzzy norm of a linear operator from a fuzzy normed linear space to another fuzzy normed linear space. In the present paper we have tried to introduce a correct definition of a fuzzy bounded linear operator and "fuzzy norm" for such an operator. With this definition of fuzzy normed linear space, it has been possible to introduce in this paper a notion of fuzzy bounded linear operator over fuzzy normed linear spaces and to define " adjoint" for such an operator.

The organization of this paper is as follows: Section 2 comprises some useful definitions, notations and preliminary results. In Section 3, the definition adjoint of the operator on fuzzy normed linear spaces is introduced and investing some important general properties of adjoint fuzzy linear operators on fuzzy normed linear spaces.

\section{Some preliminary results}

In this section some definitions and preliminary results are given which will be used in our paper. The following concepts have in $[1,4,9]$.

Definition 2. 1. A mapping $\eta: R \rightarrow[0,1]$ is called a fuzzy real number, whose $\alpha$-level set is denoted by $[\eta]_{\alpha}=\{t: \eta(t) \geq \alpha\}$, if it satisfies two axioms:

(a) There exists $t_{0} \in R$ such that $\eta\left(t_{0}\right)=1$.

(b) For each $\alpha \in(0,1]:[\eta]_{\alpha}=\left[\eta_{\alpha}^{-}, \eta_{\alpha}^{+}\right]$, where $-\infty<\eta_{\alpha}^{-} \leq \eta_{\alpha}^{+}<+\infty$. The set of all fuzzy real numbers is denoted by $F$. If $\eta \in F$ and $\eta(t)=0$ whenever $t<0$, then $\eta$ is called a non-negative fuzzy real number and $F^{+}$stands for the set of all non-negative fuzzy real numbers. The number $\overline{0}$ stands for the fuzzy number satisfying $\overline{0}(t)=1$ if $t=0$ and $\overline{0}(t)=0$ if $t \neq 0$ clearly, $\overline{0} \in F^{+}$. Since to each $r \in R$, one can consider $\bar{r} \in F$ defined by $\bar{r}(t)=1$ if $t=r$ and $\bar{r}(t)=0$ if $t \neq r, R$ can be embedded in $F$.

Definition 2.2. Let $X$ be a linear space over $R$. Let \|\|$: X \rightarrow F^{+}$be a mapping satisfying:

(i) $\|x\|=\overline{0}$ if and only if $x=0$,

(ii) $\|r x\|=|r|\|x\|, x \in X, r \in R$,

(iii) for $x, y \in X,\|x+y\| \leq\|x\| \oplus\|y\|$

And

$\left(A^{\prime}\right): x \neq 0 \Rightarrow\|x\|(t)=0, \forall t \leq 0$.

Then $(X,\|\|)$ is called a fuzzy normed linear space and \|\| is called a fuzzy norm on $X$.

Remark 2.3. (i) Condition $\left(A^{\prime}\right)$ in Definition 2.2 is equivalent to the condition $\left(A^{\prime \prime}\right)$ : for all $x(\neq 0)$ in $X,\|x\|_{\alpha}^{1}>0, \forall \alpha \in(0,1]$ where $[\|x\|]_{\alpha}=\left[\|x\|_{\alpha}^{1},\|x\|_{\alpha}^{2}\right]$ and

(ii) $\|x\|_{\alpha}^{i}: i=1,2$ are crisp norms on $X$. 
Definition 2.5. Let $(X,\|\|)$ and $\left(Y,\|\|^{\sim}\right)$ be two fuzzy normed linear spaces. An operator $T: X \rightarrow Y$ is said to be strongly fuzzy continuous at $x_{0} \in X$ if for a given $\varepsilon>0, \exists \delta>0$ such that $\left\|T x-T x_{0}\right\|_{\alpha}^{2 \sim}<\varepsilon$ whenever $\left\|x-x_{0}\right\|_{\alpha}^{1}<\delta, \forall \alpha \in(0,1]$.

If $T$ is strongly fuzzy continuous at all points of $X$, then $T$ is said to be strongly fuzzy continuous on $X$.

Definition 2.6. Let $(X,\|\|)$ and $\left(Y,\|\|^{\sim}\right)$ be two fuzzy normed linear spaces. An operator $T: X \rightarrow Y$ is said to be weakly fuzzy continuous at $x_{0} \in X$ if for a given $\varepsilon>0, \exists \delta \in F^{+}, \exists \delta \in \overline{0}$ such that

$$
\begin{aligned}
& \left\|T x-T x_{0}\right\|_{\alpha}^{\sim}<\varepsilon \text { whenever }\left\|x-x_{0}\right\|_{\alpha}^{2}<\delta_{\alpha}^{2}, \\
& \left\|T x-T x_{0}\right\|_{\alpha}^{2}<\varepsilon \text { whenever }\left\|x-x_{0}\right\|_{\alpha}^{1}<\delta_{\alpha}^{1},
\end{aligned}
$$

Where, $[\delta]_{\alpha}=\left[\delta_{\alpha}^{1}, \delta_{\alpha}^{2}\right], \alpha \in(0,1]$.

Definition 2.7. Let $(X,\|\|)$ and $\left(Y,\|\|^{\sim}\right)$ be two fuzzy normed linear spaces and $T: X \rightarrow Y$ be a linear operator. $T$ is said to be strongly fuzzy bounded if there exists a real number $k>0$ such that

$$
\|T x\| \sim \bar{k}\|x\|, \forall x(\neq 0) \in X .
$$

Notation 2.1. Denote $B(X, Y)=$ Set of all strongly fuzzy bounded linear operators defined form $(X,\|\|)$ to $\left(Y,\|\|^{\sim}\right)$.

Definition 2.8. Let $(X,\|\|)$ and $\left(Y,\|\|^{\sim}\right)$ be two fuzzy normed linear spaces and $T: X \rightarrow Y$ be a linear operator. $T$ is said to be weakly fuzzy bounded if there exists a fuzzy interval $\eta \in F^{+}, \eta>\overline{0}$ such that $\|T x\| \sim \eta\|x\|, \forall x(\neq 0) \in X$.

Remark 2.4. $T$ is strongly (weakly) fuzzy bounded linear operator from $(X,\|\|)$ to $\left(Y,\|\|^{\sim}\right)$ iff $T$ is a bounded linear operator from $\left(X,\|\|_{\alpha}^{1}\right)$ to $\left(Y,\|\|_{\alpha}^{2}\right)$ and $\operatorname{from}\left(X,\|\|_{\alpha}^{2}\right)$ to $\left(Y,\|\|_{\alpha}^{1}\right)$, $\forall \alpha \in(0,1]$.

Remark 2.5. If $T$ is strongly fuzzy bounded then it is weakly fuzzy bounded but not conversely. This is supported by the following example [1].

Notation 2.2. Denote $B^{\prime}(X, Y)=$ Set of all weakly fuzzy bounded linear operators defined form $(X,\|\|)$ to $\left(Y,\|\|^{\sim}\right)$.

Theorem 2.1. Let $T: X \rightarrow Y$ be a linear operator where $(X,\|\|)$ and $\left(Y,\|\|^{\sim}\right)$ are two fuzzy normed linear spaces. Then $T$ is strongly (weakly) fuzzy continuous iff it is strongly (weakly) fuzzy bounded 
Theorem 2.2. A linear operator $T$ from $(X,\|\|)$ and $\left(Y,\|\|^{\sim}\right)$ is strongly (weakly) fuzzy continuous iff it is strongly (weakly) fuzzy continuous at a point.

Theorem 2.3. The set $B(X, Y)\left(B^{\prime}(X, Y)\right)$ of all strongly (weakly) bounded linear operators from a fuzzy normed linear space $(X,\|\|)$ to a fuzzy normed linear space $\left(Y,\|\|^{\sim}\right)$ is a linear space with respect to usual linear operators.

Definition 2.9. A strongly (weakly) fuzzy bounded linear operator defined from a fuzzy normed linear space $(X,\|\|)$ to $\left(R,\|\|^{\sim}\right)$, is called a strongly (weakly) fuzzy bounded linear functional. Where, the function $\|r\|^{\sim}: R \rightarrow[0,1]$ is defined by

$$
\|r\| \sim(t)= \begin{cases}1 & \text { if } t=|r| \\ 0 & \text {, otherwise }\end{cases}
$$

Then $\|r\|^{\sim}$ is a fuzzy norm on $R$ and $\alpha$-level sets of $\|r\|^{\sim}$ are given by $\left[\|r\|^{\sim}\right]_{\alpha}=[|r|,|r|], 0<\alpha \leq 1$. Denote by $X^{*}\left(X^{\prime}\right)$ the set of all strongly (weakly) fuzzy bounded linear functionals over $(X,\|\|)$. We call $X^{*}\left(X^{\prime}\right)$ the first strong (weakly) fuzzy dual space of $X$.

\section{The main results}

In this section Hahn- Banach theorem is given which will be used in our main results.

Theorem 3.1. (Hahn- Banach [1]) . Let $(X,\|\|)$ be a fuzzy normed linear space and $Z$ be a subspace of $X$. Let $f$ be a strongly fuzzy bounded linear functional defined on $(Z,\|\|)$. Then there exists a strongly fuzzy bounded linear functional $\Lambda$ on $X$ such that $\left.\Lambda\right|_{Z}=f$ and $\|f\|_{Z}^{*} \leq\|\Lambda\|_{X}^{*}$.

Lemma 3.1. [2]. Let $(X,\|\|)$ bea a fuzzy normed linear space and $x_{0} \in X$, then the exists a strongly fuzzy bounded linear functional $\Lambda \in X^{*}$ such that $\|\Lambda\|_{\alpha}^{*}=\overline{1}$ and $\Lambda x_{0}=\left\|x_{0}\right\|_{\alpha}, \forall \alpha \in(0,1]$.

Definition 3.1. Let $(X,\|\|)$ and $\left(Y,\|\|^{\sim}\right)$ are two fuzzy normed linear spaces and $T \in B(X, Y)\left(B^{\prime}(X, Y)\right)$. Operator $T^{*}: Y^{*} \rightarrow X^{*}$ is defined

$$
\left(T^{*} \Lambda\right)(x)=\Lambda(T x), \Lambda \in Y^{*}, x \in X .
$$

Operator $T^{*}$ is called adjoint operator $T$.

Theorem 3.2. Let $(X,\|\|)$ and $\left(Y,\|\|^{\sim}\right)$ are two fuzzy normed linear spaces. If $T \in B(X, Y)$ then $T^{*} \in B\left(Y^{*}, X^{*}\right)$ and $\|T\|_{\alpha}^{*}=\left\|T^{*}\right\|_{\alpha}^{*}, \forall \alpha \in(0,1]$. 
Proof. For each $\Lambda \in Y^{*}$ we get $T^{*} \Lambda=\Lambda T$ and hence $T^{*} \Lambda$ is linear and continuous. So for each $\Lambda \in Y^{*}$ we have $T^{*} \Lambda \in X^{*}$. Clearly, $T^{*}$ is linear. Since $T$ is strongly fuzzy bounded linear, the exists $k(>0) \in R$ such that

$$
\|T x\| \sim \bar{k}\|x\|, \quad \forall x(\neq 0) \in X,
$$

i.e.

$$
\begin{aligned}
& \frac{\|T(x)\|_{\alpha}^{1}}{\|x\|_{\alpha}^{2}} \leq k \text { and } \frac{\|T(x)\|_{\alpha}^{2}}{\|x\|_{\alpha}^{1}} \leq k \quad \forall x(\neq 0) \in X . \text { We define } \\
& \|T\|_{\alpha}^{* 1}=\sup _{x \in X, x \neq 0} \frac{\|T x\|_{\alpha}^{-1}}{\|x\|_{\alpha}^{2}}(\leq k), \\
& \|T\|_{\alpha}^{* 2}=\sup _{x \in X, x \neq 0} \frac{\|T x\|_{\alpha}^{\sim 2}}{\|x\|_{\alpha}^{1}}(\leq k) .
\end{aligned}
$$

Hence

$$
\left.\begin{array}{l}
\|T x\|_{\alpha}^{\sim 1} \leq\|T\|_{\alpha}^{* 1}\|x\|_{\alpha}^{2} \\
\|T x\|_{\alpha}^{\sim 2} \leq\|T\|_{\alpha}^{* 2}\|x\|_{\alpha}^{1}
\end{array}\right\}, \forall \alpha \in(0,1], \forall x \in X
$$

Then $\left\{\|\|_{\alpha}^{* 1}: \alpha \in(0,1]\right\}$ and $\left\{\|\|_{\alpha}^{* 2}: \alpha \in(0,1]\right\}$ are, respectively, ascending and descending families of norms. Also from above it follows that $\left\{\left[\|T\|_{\alpha}^{* 1},\|T\|_{\alpha}^{* 2}\right]: 0<\alpha \leq 1\right\}$ is a family of nested bounded closed intervals of real numbers.

Let $x \in X$ and $\Lambda \in Y^{*}$, we have

$$
\left\|\left(T^{*} \Lambda\right)(x)\right\|_{\alpha}^{* 1}=\|\Lambda(T x)\|_{\alpha}^{* 1} \leq|\Lambda|\|T x\|_{\alpha}^{\sim 1} \leq \mid \Lambda\|T\|_{\alpha}^{* 1}\|x\|_{\alpha}^{2}, \quad \forall \alpha \in(0,1],
$$

So

$T^{*}$ continuous and $\left\|T^{*}\right\|_{\alpha}^{* 1} \leq\|T\|_{\alpha}^{* 1}, \forall \alpha(0,1], \quad(i i)$.

For $\varepsilon>0$, so $\|T\|_{\alpha}^{*}$ implies that there exists a $x$ such that $\|x\|_{\alpha}=1$ and $\|T x\|_{\alpha}^{\sim 1}>\|T\|_{\alpha}^{* 1}-\varepsilon$ By Lemma 3.1 the exists $\Lambda \in Y^{*}$ such that $\|\Lambda\|_{\alpha}^{* 1}=1$ and $\Lambda(T x)=\|T x\|_{\alpha}^{\sim 1}$.

$$
\left|\left(T^{*} \Lambda\right)(x)\right|=|\Lambda(T x)|=\|T x\|_{\alpha}^{\sim 1}>\|T\|_{\alpha}^{* 1}-\varepsilon, \forall \alpha \in(0,1],
$$

Thus

$$
\left\|T^{*}\right\|_{\alpha}^{* 1} \geq\left\|T^{*} \Lambda\right\|_{\alpha}^{* 1}>\|T\|_{\alpha}^{* 1}-\varepsilon, \forall \alpha \in(0,1],
$$

So

$\left\|T^{*}\right\|_{\alpha}^{* 1} \geq\|T\|_{\alpha}^{* 1}, \forall \alpha \in(0,1], \quad$ (iii)

(ii) and (iii) imply that 


$$
\left\|T^{*}\right\|_{\alpha}^{* 1}=\|T\|_{\alpha}^{* 1}, \forall \alpha \in(0,1],
$$

Similarly we have

$$
\left\|T^{*}\right\|_{\alpha}^{* 2}=\|T\|_{\alpha}^{* 2}, \forall \alpha \in(0,1]
$$

So

$$
\|T\|_{\alpha}^{*}=\left\|T^{*}\right\|_{\alpha}^{*}, \forall \alpha \in(0,1]
$$

Theorem 3.3. Let $(X,\|\|)$ and $\left(Y,\|\|^{\sim}\right)$ be two fuzzy normed linear spaces. If $T \in B^{\prime}(X, Y)$ then $T^{*} \in B^{\prime}\left(Y^{*}, X^{*}\right)$ and $\|T\|_{\alpha}^{*}=\left\|T^{*}\right\|_{\alpha}^{*}, \forall \alpha \in(0,1]$.

Proof. The proof is similar to Theorem 3.2 and therefore, it is omitted.

\section{References}

[1] Bag, T., and Samanta, S. K., "Fuzzy bounded linear operators in Felbin's type fuzzy normed linear spaces", Fuzzy Sets and Systems, Vol. 159, pp. 685 - 707, 2008.

[2] Bag, T., and Samanta, S. K., "Fuzzy bounded linear operators", Fuzzy Sets and Systems Vol. 151, pp. 513-547, 2005.

[3] Cheng, S. C., and Mordeson, J. N., "Fuzzy linear operator and fuzzy normed linear spaces", Bull. Cal. Math., Soc. Nol. 86, pp. 429-436, 1994.

[4] Felbin, C., "Finite dimensional fuzzy normed linear space", Fuzzy Sets and Systems, Vol. 48 pp. 239-248, 1992.

[5] Felbin, C., "Finite dimensional fuzzy normed linear spaces II", J. Analysis 7 (1999) 117131.

[6] Kaleva, O., and Seikkala, S., "On fuzzy metric spaces", Fuzzy Sets and Systems, Vol. 12, pp. 215-229, 1984.

[7] Katsaras, A. K., "Fuzzy topological vector spaces II", Fuzzy Sets and Systems, Vol. 12, pp. 143-154, 1984.

[8] Kramosil, I., and Michelek, J., "Fuzzy metric and statistical metric spaces", Kybernetica, Vol. 11, pp. 326-334, 1975.

[9] Xiao, J. Z., and Zhu, X. h., "Fuzzy normed spaces of operators and its completeness", Fuzzy Sets and Systems, Vol. 133 No.3, pp. 389-399, 2003. 